Original Article

\title{
Comparative evaluation of rapid Salmonella Typhi IgM/IgG and Widal test for the diagnosis of enteric fever
}

\author{
Farjana Akter ${ }^{1}$, Mahmuda Yeasmin ${ }^{2}$, Md. Zahangir Alam ${ }^{3}$, Md. Rokibul Hasan ${ }^{4}$, Fahmida Rahman ${ }^{1}$, Elisha \\ Khandker $^{1}$, Md. Monirul Hoque ${ }^{1}$, Lovely Barai ${ }^{4}$, Md. Mohiuddin ${ }^{1}$, Md. Shariful Alam Jilani ${ }^{1}$ \\ ${ }^{1}$ Department of Microbiology, Ibrahim Medical College, Dhaka, Bangladesh; ${ }^{2}$ Department of Virology, \\ Bangabandhu Sheikh Mujib Medical University (BSMMU), Dhaka, Bangladesh; ${ }^{3}$ Bangladesh Specialized \\ Hospital, Dhaka, Bangladesh; ${ }^{4}$ Department of Microbiology, Bangladesh Institute of Research and \\ Rehabilitation in Diabetes, Endocrine and Metabolic Disorders (BIRDEM), Dhaka, Bangladesh
}

\begin{abstract}
Background: Accurate and early diagnosis of enteric fever is a diagnostic challenge where facility for blood culture is not available. As a result, Widal test is still used widely in resource limited settings. Recently, user-friendly rapid immunochromatographic tests (ICT) have been introduced for quick diagnosis of enteric fever. So, we evaluated sensitivity and specificity of an immunochromatography based Salmonella Typhi IgM/IgG test kit and Widal test compared to blood culture for the diagnosis of enteric fever.
\end{abstract}

Method: The study was conducted in the Department of Microbiology, Ibrahim Medical College (IMC) and Bangladesh Institute of Research and Rehabilitation in Diabetes, Endocrine and Metabolic Disorders (BIRDEM) from June 2017 to September 2017. Clinically suspected enteric fever cases were included. Blood culture, Widal and Salmonella Typhi IgM/IgG detecting ICT were employed for the diagnosis of enteric fever.

Results: Out of 71 suspected cases of enteric fever, blood culture was positive in 36 cases (50.7\%) while $42(59.15 \%)$ and 35 (49.29\%) cases were positive by Widal test and ICT respectively. Widal and ICT had sensitivity and specificity of $100 \%$ and $89.9 \%$ and $82.9 \%$ \& $91.4 \%$ respectively.

Conclusion: Findings of the study suggest that both Widal and immunochromatographic tests can be used interchangeably for rapid diagnosis of enteric fever.

IMC J Med Sci 2020; 14(1): 004. EPub date: 29 February 2020

\section{Introduction}

Enteric fever is a multisystem disease and its outcome can be fatal if not properly diagnosed and treated [1]. It is predominantly caused by Salmonella enterica serotype Typhi and less frequently by Salmonella enterica serotype Paratyphi $A$ and $B$ [2]. Lack of access to safe drinking water, unhygienic sanitation, and overcrowded population of underdeveloped countries may accelerate its fecooral transmission [3]. Physicians often experience diagnostic dilemma due to its protean clinical presentation which is quite similar to other febrile illness like dengue, malaria, chikungunya etc. in endemic area [4]. Prompt and accurate diagnosis of enteric fever is a pressing need albeit no such diagnostic test is currently available that can provide $100 \%$ sensitivity and accuracy. Diagnosis at an early stage can reduce indiscriminate antibiotic use; prevent unwanted life threatening complications and chronic carrier state [5]. Amidst available

\section{Address for Correspondence:}

Dr. Farjana Akter. Lecturer, Department of Microbiology, Ibrahim Medical College, 1/A Ibrahim Sarani, Segunbagicha, Dhaka-1000, Bangladesh, 10 ${ }^{\text {th }}$ floor, Room: 1118. Email: farjana73mail@yahoo.com 
diagnostic test, isolation of organisms from blood, bone marrow, urine and stool is considered gold standard for diagnosis of enteric fever $[6,7]$. Culturing of organism from blood is frequently done in clinical setting which is insufficiently sensitive, laborious and time consuming and bone marrow culture, although more sensitive is not done routinely due to its high technical demand $[8,9]$. In spite of considering blood culture as a gold standard test, it is not available in every primary health care setting. Moreover, its turnaround time is longer, usually 2-3 days. As a result, diagnosis of enteric fever overlooked or delayed and based on clinical features, clinicians often provide unnecessary antimicrobial therapy or undertreat the patients when other differentials are considered [10,11]. Therefore, rapid, simple, convenient, easy to perform, sensitive serological test to identify Salmonella Typhi and Paratyphi is often considered as the only diagnostic tool that can guide clinicians [12].

Most routinely performed serological test is Widal which was developed by Georges Fernand Widal in 1890 based on the demonstration of agglutinating antibodies against lipopolysaccharide (LPS; O) and flagella $(\mathrm{H})$ antigens of Salmonella Typhi and Paratyphi A and B. This test became obsolete in many developed countries due to its unsatisfactory results, low prevalence of enteric fever and availability of more sophisticated diagnostic tools [13]. Variable sensitivity and specificity of Widal test was documented in different studies and its role as a diagnostic tool is still debatable. However, some studies conducted in Tanzania, Vietnam, Bangladesh, India during different periods of time stated that Widal test could be relevant as a diagnostic tool and could be an alternative to blood culture [14-17]. On the other hand, findings of other studies conducted in Pakistan, Nepal, South Africa, Tanzania, and Ethiopia indicated that Widal test alone might not be suitable to diagnose enteric fever as it could produce false positive results [18]. But scenario is quite different in developing countries like Bangladesh where Widal test is still used widely as facility of culturing organism is limited to only in tertiary care hospitals, lack of trained personnel and prohibitively high cost of culture compared to serological test [16].

User friendly rapid diagnostic tests (RDT) for diagnosis of Salmonella Typhi are available commercially in different methods and formats like ELISA or immunochromatography based tests (ICT) which can directly detect IgM and/or IgG antibodies against specific antigen of Salmonella Typhi [19]. It can also detect antibodies within 4-5 days of appearance of fever and ICT can provide results within 15-30 minutes. ICT is user-oriented, time saving and does not require highly skilled personnel to perform the test and to interpret the result which makes it an excellent choice for point of care service [20]. But these kits are still not widely acceptable due to its inconsistent sensitivity (7395\%) and specificity (68-95\%) which have been documented in different studies conducted preliminarily in different Asian countries [21-25].

Therefore, the aim of the current study was to evaluate the sensitivity and specificity of both Widal test and rapid Salmonella Typhi IgM/IgG immunochromatographic test in comparison to blood culture for quick and accurate diagnosis of typhoid fever.

\section{Materials \& Methods}

Study population, place and samples collection: This study was conducted in the Department of Microbiology, Ibrahim Medical College (IMC) and Bangladesh Institute of Research and Rehabilitation in Diabetes, Endocrine and Metabolic Disorders (BIRDEM) from June 2017 to September 2017. Total 71 blood samples were collected from suspected cases of enteric fever for blood culture, Widal test and Salmonella Typhi IgM/IgG immunochromatographic test. From each patient $10 \mathrm{ml}$ of venous blood was collected aseptically for blood culture and serological tests. Informed written consent was obtained from each patient prior to collection of blood.

Blood culture: An aliquot $(8 \mathrm{ml}$ for adult and $1.5 \mathrm{ml}$ for children) of fresh blood was immediately processed for culture. Blood culture was done by lysis centrifugation method and inoculated on blood agar and MacConkey agar media and incubated for 48 hours at $37^{\circ} \mathrm{C}[25,26]$. Suspected bacterial colony was identified by Gram staining and standard biochemical tests $[25,27]$. Serotype of Salmonella spp. was identified by slide agglutination test by specific 'O' (lipopolysaccharide), ' $\mathrm{H}$ ' (flagella) antisera [27]. 
Widal test and Salmonella IgM/IgG ICT: Widal test was carried out by slide method using $\mathrm{HiPer}^{\circledR}$ Widal Test Teaching Kit (HiMedia Laboratories Pvt. Limited, India) according to the manufacturer's instruction. Test results were interpreted visually by demonstrating agglutinating antibody titres against ' $\mathrm{O}$ ' (lipopolysaccharide) and ' $\mathrm{H}$ ' antigen (flagella) of Salmonella spp. An antibody titre of $1: 80$ or more against ' $\mathrm{O}$ ' and ' $\mathrm{H}$ ' antigen was considered positive [28].

Enteroscreen-WB ICT kit Typhi manufactured by Zephyr Biomedical (Verna Industrial Estate, Verna, Goa, India) was used to detect Salmonella IgM/IgG antibodies against an outer membrane protein of Salmonella Typhi. Test was carried out as per manufacturer's instruction and reading was taken after 15-30 minutes based on appearance of coloured band in the control region and test region. The band in test region represented presence of either anti-Salmonella IgM or IgG. ICT was considered positive if any anti-Salmonella IgM, IgG or IgM+lgG band appeared positive in any sample. The result was compared with blood culture and Widal test.

\section{Results}

Total 71 suspected cases of enteric fever were included in the study. Out of 71 cases, blood culture, Widal and ICT were positive in 36 (50.7\%), $42(59.2 \%)$ and $35(49.3 \%)$ cases respectively (Table-1). Out of 36 blood culture positive cases, Salmonella Typhi was isolated from 32 cases and Salmonella Paratyphi A was present in 4 cases (Table-2).

Widal test was positive in all $S$. Typhi and $S$. Paratyphi A positive cases. Out of 35 culture negative cases, 6 cases were Widal test positive as well. Widal test showed more than 1:80 titre of $\mathrm{TO} / \mathrm{TH}$ in all $\mathrm{S}$. Typhi culture positive cases and higher titre of 'AO'/'AH' was observed in all $S$. Paratyphi A cases. Although TO/TH is specific to $S$. Typhi, higher titre was also observed in all culture positive cases of $S$. Paratyphi $A$ and at the same time titre of $\mathrm{AO} / \mathrm{AH}$ which was specific to $S$. Paratyphi A was raised in 11 cases of S. Typhi. ICT for Salmonella IgM/IgG was performed in all 71 cases. ICT was positive in total $32(88.9 \%)$ out of 36 blood culture positive cases. Out of $32 \mathrm{~S}$. Typh positive cases, 29 cases were positive by ICT and 3 were negative by ICT. On the other hand among 4 S. Paratyphi A positive cases, 3 showed positive result in ICT (Table-2).

Table-1: Results of blood culture, Widal and Salmonella-IgM/IgG ICT tests $(n=71)$

\begin{tabular}{lcc}
\hline Test & $\begin{array}{c}\text { Positive } \\
\text { Number (\%) }\end{array}$ & $\begin{array}{c}\text { Negative } \\
\text { Number (\%) }\end{array}$ \\
\hline $\begin{array}{l}{ }^{\mathrm{a} B l o o d} \text { culture } \\
\text { for Salmonella } \\
\text { spp. }\end{array}$ & $36(50.7 \%)$ & $35(49.3 \%)$ \\
Widal Test & $42(59.2 \%)$ & $30(40.84 \%)$ \\
Salmonella- & $35(49.3 \%)$ & $36(50.71 \%)$ \\
IgM/IgG ICT & & \\
\hline Note: $a=$ includes S. Typhi and S. Paratyphi A
\end{tabular}

Only anti-Salmonella IgM, IgG and both $\operatorname{lgM}$ and IgG were positive in 3 (4.2\%), 18 (25.4\%) and 14 (19.7\%) cases respectively (Table-3). Total 32 cases (45.1\%) were IgG positive, 17 (23.9\%) were IgM positive and 14 (29.17\%) were both IgM and IgG positive (Table-3). The sensitivity and specificity of Widal and Salmonella IgM/IgG ICT are shown in Table-4. The sensitivity and specificity of Widal test were $100 \%$ and $82.9 \%$ respectively while these were $88.9 \%$ and $91.4 \%$ for Salmonella IgM/IgG ICT. Salmonella IgM/IgG test had higher (91.4\%) positive predictive value (PPV) compared to Widal test $(85.7 \%)$.

Table-2: Comparative results of blood culture, Widal test and Salmonella-IgM/IgG ICT ( $n=71)$

\begin{tabular}{lccccc}
\hline \multirow{2}{*}{$\begin{array}{l}\text { Blood culture } \\
\text { result }\end{array}$} & \multirow{2}{*}{ No } & \multicolumn{2}{c}{ Widal test } & \multicolumn{2}{c}{ ICT for Salmonella-IgM/IgG } \\
\cline { 3 - 6 } & & Positive & Negative & Positive & Negative \\
\hline S. Typhi & 32 & 32 & 0 & 29 & 3 \\
S. Paratyphi A & 4 & 4 & 0 & 3 & 1 \\
Negative & 35 & 6 & 29 & 3 & 32 \\
\hline Total & 71 & $42(59.2 \%)$ & $29(40.8 \%)$ & $35(49.3 \%)$ & $36(50.7 \%)$ \\
\hline
\end{tabular}


Table-3: Rate and pattern of Salmonella $\operatorname{lgM} / \lg G$ antibodies by ICT in study cases $(n=71)$

\begin{tabular}{lc}
\hline ICT & $\begin{array}{c}\text { Positive Number } \\
\text { (\%) }\end{array}$ \\
\hline Only IgM & $3(4.2 \%)$ \\
Only IgG & $18(25.4 \%)$ \\
Both IgG and IgM & $14(19.7 \%)$ \\
\hline Note: Total IgM positive: $17(23.9 \%) ;$ Total IgG \\
positive: 32 (45.1\%).
\end{tabular}

Table-4: Sensitivity, specificity, PPV and NPV of Widal and Salmonella IgM/lgG tests

\begin{tabular}{lcccc}
\hline Test & Sensitivity & Specificity & PPV & NPV \\
\hline Widal & $100 \%$ & $82.9 \%$ & $85.7 \%$ & $100 \%$ \\
Salmonella & $88.9 \%$ & $91.4 \%$ & $91.4 \%$ & $88.9 \%$ \\
IgM/IgG ICT & & & & \\
\hline Note: PPV: Positive predictive value; NPV: Negative \\
predictive value.
\end{tabular}

\section{Discussion}

Isolation of Salmonella Typhi and Paratyphi A and B from blood for diagnosis of enteric fever is the current recommendation of WHO and considered as a reference while evaluating other tests [29]. Blood culture is highly specific but its suboptimal sensitivity after the first week of illness leads to the diagnostic difficulty and sensitivity [30]. In this study, it has been observed that blood culture for Salmonella enterica serotype Typhi and Paratyphi A was found to be positive in $50.7 \%$ cases which is quite similar to other study findings where $40-70 \%$ of presumptive cases were found culture positive [7,31-35]. In contrast to these study findings, rate of isolation of Salmonella spp. was found much lower ranging from $8.9-43 \%$ in many well documented studies [36-39]. This low rate of isolation may be attributed to negligence of seeking health care services at an early stage of fever, inappropriate use of antibiotic before blood culture and collection of inadequate amount of blood especially in case of children [40].

In this current series, both Widal test and ICT (Enteroscreen-WB) were performed in 71 clinically suspected cases of enteric fever to evaluate their sensitivity, specificity, positive predictive value
(PPV) and negative predictive value (NPV) compared to blood culture. Out of 42 Widal positive cases 36 cases were blood culture positive and 6 cases were blood culture negative. Sensitivity, specificity, PPV and NPV of Widal were noted as $100 \%, 82.9 \%, 85.7 \%$ and $100 \%$ respectively. In this study, the sensitivity of Widal was found higher though specificity was slightly reduced and was in accordance with the reported results of blood culture. Gopala kirshnan et al. [41] in 2002 reported sensitivity and specificity of Widal test as $98 \%$ and $76 \%$ respectively which closely resemble our study findings. Another study reported the sensitivity and specificity of Widal test as $71 \%$ and $62 \%$ [17]. In 2016, a study from Bangladesh reported the sensitivity, specificity, PPV and NPV of Widal test as $83.3 \%, 80 \%, 86.2 \%$, and $76.2 \%$ respectively [42]. These study findings revealed fairly good diagnostic accuracy of Widal test for diagnosis of enteric fever.

In the present study raised titre of TO/TH of more than 1:80 was observed in all Salmonella Paratyphi A positive cases and $\mathrm{AO} / \mathrm{AH}$ in 11 Salmonella Typhi positive cases. This may be due to cross reacting antigen between these serotypes. Moreover, lipopolysaccharide ' $O$ ' antigen also shared by other Enterobacteriaceae which results in false positive Widal test making the test less specific to detect Salmonella spp.

Several studies have claimed that rapid diagnostic tests (RDT) provide better valid results than Widal test with regard to sensitivity and specificity [22, 43]. In our study with lateral flow rapid Salmonella IgM/IgG ICT, the sensitivity, specificity, PPV and NPV were recorded as $88.89 \%, 91.43 \%, 91.43 \%$ and $88.89 \%$ respectively. According to a study done by Sanjeev et al. [44], Typhi-dot performed better than Widal test and they found sensitivity and specificity of Widal and Typhi-dot as $100 \%$ and $76 \%$ and $78.78 \%$ and $58.82 \%$ respectively. They suggested that rather than using Widal test it might be more useful to use rapid test like Typhi-dot in routine diagnostic service besides blood culture. Studies from Bangladesh reported similar rate of sensitivity and specificity of a rapid ICT (SD Bioline) and TUBEX $^{\circledR}$ for the diagnosis of typhoid fever $[45,46]$. This is in agreement with our findings. In contrary to these findings, Neheed et al. 
demonstrated suboptimal performance of Typhidot and TUBEX ${ }^{\circledR}$ to diagnose typhoid fever among community populations [47]. Dissimilarity regarding the sensitivity and specificity observed in different studies could be due to the use of different format of rapid diagnostic test kit from different manufacturers. In addition, time elapsed from onset of symptoms and performance of test may affect sensitivity and specificity of ICT.

Among the available different RDT kit, diagnostic accuracy of Typhi-dot and TUBEX ${ }^{\circledR}$ were largely studied. Very limited studies were conducted where performance of Enteroscreen ICT (Bioline) was analysed. Prasad et al. included 2699 patients in their study to compare the diagnostic validity of two rapid Salmonella IgM tests with regard to blood culture [48]. Sensitivity, specificity, PPV and NPV of Typhi-dot and Enteroscreen were recorded as $97.29 \%, 97.40 \%, 98.18 \%$ and $96.15 \%$ and $88.13 \%, 87.83 \%, 92.03 \%$ and $82.27 \%$ respectively. Though Enteroscreen performed poorly in comparison to Typhi-dot, they recommend Enteroscreen during emergency situation due to its acceptable PPV and it takes less time to provide results [48]. Our data matched well with these values. On the other hand, another study mentioned sensitivity, specificity, PPV and NPV of Enteroscreen ICT in comparison to Widal as gold standard test as 50\%, 96\%, 66.66\% and $92.30 \%$ respectively. Specificity and NPV of ICT were similar to our study while they found poor sensitivity and PPV of this particular ICT kit [49]. Although this ICT kit is meant to detect $S$. Typhi only, but in this study we observed that out of $4 \mathrm{~S}$. Paratyphi A cases, 3 (75\%) were positive by this kit. This could be attributed to cross reactivity between outer membrane protein of both Salmonella spp. Prasad et al. (2015) noted Enteroscreen ICT positive results in 22 cases of 46 blood culture positive $S$. Paratyphi cases and sensitivity was $47.83 \%$ in their study [48]. This cross reactivity provides extra advantages of diagnosing paratyphoid fever by Enteroscreen ICT kit.

The ICT kit that we have used is able to differentiate between IgM and IgG antibodies. This study demonstrated that only IgM became positive in $4.2 \%$ cases, only IgG in $25.4 \%$ cases and both IgM and IgG was found positive in $19.7 \%$ cases. Only
IgM (early phase) or both IgM and IgG positive (middle phase) indicates current infection and IgG without IgM usually denotes past, reinfection or late stage disease when sero-conversion has already been occurred. In this study we observed the percentages of IgG positive cases among culture positive group was high, but in other studies conducted through different ICT kit found more IgM positive cases [45]. This could be explained by the disappearance of $\operatorname{lgM}$ in the late stage of disease or masking of IgM by IgG [50].

In our study, Enteroscreen Salmonella IgM/IgG rapid test showed satisfactory results in terms of sensitivity and specificity compared to Widal test. Our results differ to some extent from above mentioned studies and this might be due to small sample size and collection of blood at different stage of fever. So, these serological tests may be used interchangeably with Widal test in suspected enteric fever where an adequate laboratory facility for blood culture is not available.

Development of convenient, rapid, highly sensitive, specific and robust diagnostic tool is a long felt need to diagnose enteric fever accurately at an early stage of disease. In this regard, rapid serological diagnostic tests in ICT format with considerable sensitivity and specificity can play a fundamental role, especially in resourceconstrained rural settings of Bangladesh. At the same time, usefulness of Widal test in area with limited microbiology laboratory facilities cannot be ignored. Although clinical implication of Widal test is reducing day by day, but in some areas, it is the only available test in which clinicians have to rely to reach a diagnosis of enteric fever. Our work has led us to conclude that Widal test is justifiable as long as the results are interpreted in accordance with the clinical history indicative of enteric fever and background level of antibody titres of local populations are considered. At the same time introduction of ICT might be an important addition to serological test for more rapid and reliable diagnosis of enteric fever.

\section{Acknowledgement}

We are grateful to Tradesworth Ltd. Bangladesh for providing Enteroscreen-WB ICT kit. 


\section{References}

1. Nasrallah SM, Nassar VH. Enteric fever: a clinicopathologic study of 104 cases. Am J Gastroenterol. 1978; 69(1): 63-69.

2. Joshi S. Antibiogram of $S$. enterica serovar typhi and S. enterica serovar paratyphi A: a multi-centre study from India. WHO South East Asia J Public health. 2012; 1(2): 182-183.

3. Corner RJ, Dewan AM, Hashizume $M$. Modelling typhoid risk in Dhaka metropolitan area of Bangladesh: the role of socio-economic and environmental factors. Int J Health Geogr. 2013; 12: 13.

4. Bhutta ZA, Dewraj HL. Current concepts in the diagnosis and treatment of typhoid fever. BMJ. 2006; 333(7558): 78-82.

5. Baker S, Favorov M, Dougan G. Searching for the elusive typhoid diagnostic. BMC Infect Dis. 2010; 10: 45.

6. Gasem $\mathrm{MH}$, Dolmans WM, Isbandrio $\mathrm{B}$, Wahyono $\mathrm{H}$, Keuter M, Djokomoeljanto $\mathrm{R}$. Culture of Salmonella Typhi and Salmonella Paratyphi from blood and bone marrow in suspected typhoid fever. Trop Geogr Med. 1995; 47(4): 164-167.

7. Gilman $\mathrm{RH}$, Terminel $\mathrm{M}$, Levine $\mathrm{MM}$, Hernandez-Menodoze $\mathrm{P}$, Hornick Rb. Relative efficacy of blood, urine, rectal swab, bone marrow and rose spot cultures for recovery of Salmonella Typhi in typhoid fever. Lancet. 1975; B(7918): 1211-1213.

8. Hoffman SL, Edman DC, Punjabi NH, Lesmana M, Cholid A, Sundah S, et al. Bone marrow aspirate culture superior to streptokinase clot culture and $8 \mathrm{ml} \mathrm{1:} 10$ blood-to-broth ratio blood culture for diagnosis of typhoid fever. Am J Trop Med Hyg. 1986; 35(4): 836-839.

9. Parry CM, Wijedoru L, Arjyal A, Baker S. The utility of diagnostic tests for enteric fever in endemic locations. Expert Rev Anti Infect Ther. 2011; 9(6): 711-725.

10. Ross IN, Abraham T. Predicting enteric fever without bacteriological culture results. Trans $R$ Soc Trop Med Hyg. 1987; 81(3): 374-377.
11. Archibald LK, Reller LB. Clinical microbiology in developing countries. Emerg Infect Dis. 2011; 7: 302-305.

12. House D, Wain J, Ho VA, Diep TS, Chinh NT, Bay $P V$, et al. Serology of typhoid fever in an area of endemicity and its relevance to diagnosis. $J$ Clin Microbiol. 2001; 39(3): 1002-1007.

13. Olopoenia LA, King AL. Widal agglutination test - 100 years later: still plagued by controversy. Postgrad Med J. 2000; 76(892): 80-84.

14. Ley B, Mtove G, Thriemer K, Amos B, Von Seidlein L, Hendriksen I, et al. Evaluation of the Widal tube agglutination test for the diagnosis of typhoid fever among children admitted to a rural hospital in Tanzania and a comparison with previous studies. BMC Infect. Dis. 2010; 10(1): 180.

15. Parry CM, Hoa NT, Diep TS, Wain J, Chinh NT, Vinh $\mathrm{H}$, et al. Value of a single-tube Widal test in diagnosis of typhoid fever in Vietnam. J Clin Microbiol. 1999; 37(9): 2882-2886.

16. Alam AS, Rupam FA, Chaiti F. Utility of a single Widal test in the diagnosis of typhoid fever. Bangladesh J Child Health. 2011; 35(2): 53-58.

17. Aziz T, Haque SS. Role of Widal test in the diagnosis of typhoid fever in context to other test. Am J Biochem. 2012; 2(1): 16-18.

18. Mengist HM, Tilahun K. Diagnostic value of Widal test in the diagnosis of typhoid fever: a systematic review. J Med Microbiol Diagn. 2017; 6: 248.

19. Prasad KJ, Oberoi JK, Goel N, Wattal C. Comparative evaluation of two rapid Salmonella-IgM tests and blood culture in the diagnosis of enteric fever. Indian J Med Microbiol. 2015; 33(2): 237-242. [Letter]

20. Wijedoru L, Mallett S, Parry CM. Rapid diagnostic tests for typhoid and paratyphoid (enteric) fever. Cochrane Database Syst Rev. 2017; 5: CD008892.

21. Sherwal BL, Dhamija RK, Randhawa VS, Jais $M$, Kaintura A, Kumar M. A comparative study of Typhidot $M$ and Widal test in patients of typhoid fever. J Indian Acad Clin Med. 2004; 5(3): 244-260. 
22. Jesudason MV, Sivakumar S. Prospective evaluation of a rapid diagnostic test Typhidot for typhoid fever. Indian J Med Res 2006; 123(4): 513-516.

23. Choo KE, Davis TM, Ismail A, Ibrahim TA, Ghazali WN. Rapid and reliable serological diagnosis of enteric fever: Comparative sensitivity and specifcity of Typhidot and Typhidot-M tests in febrile Malaysian children. Acta Trop. 1999; 72: 175-183.

24. Bhutta ZA, Mansurrali N. Rapid serologic diagnosis of pediatric typhoid fever in an endemic area: A prospective comparative evaluation of two dot enzyme immunoassay and the Widal test. Am J Trop Med Hyg. 1999; 61(4): 654-657.

25. Cheesbrough M. District Laboratory Practice in Tropical Countries. $2^{\text {nd }}$ ed. India: Cambridge University Press; 2012. p. 184-186.

26. Sultana S, Hossain MA, Al Maruf MA, Gani MA. Comparison of the lytic blood culture method with the conventional blood culture method in cases of enteric fever in a tertiary care hospital. Bangladesh J Infect Dis. 2016; 3(1): 6-10.

27. Baron E. Specimen collection, transport and processing: Bacteriology. In: Jorgensen $\mathrm{JH}$, Pfaller MA, Carroll KC, Funke G, Landry ML, Richter SS, Warnock DW, editors. Manual of Clinical Microbiology. $11^{\text {th }}$ eds. Washington, DC: ASM Press; 2007; p310.

28. Khan A, Ashher F, Khanam F, Rahman MM, Khan M, Paul SK, Hossain MA. Baseline Widal titer among healthy adult males from the greater Mymensingh division of Bangladesh. Infect Disord Drug Targets. 2018; 18(3): 233 240.

29. World Health Organisation. The diagnosis, treatment and prevention of typhoid fever; Communicable disease surveillance and response vaccine and biologicals. Geneva: World Health Organisation; 2003. 38p.

30. Buckle GC, Walker CLF, Black RE. Typhoid fever and paratyphoid fever: Systematic review to estimate global morbidity and mortality for 2010. J Glob Health. 2012; 2(1): 010401.
31. Farooqui $B J$, Khurshid M, Ashfaq MK, Khan MA. Comparative yield of Salmonella Typhi from blood and bone marrow cultures in patients with fever of unknown origin. J Clin Pathol. 1991; 44(3): 258-259.

32. Ochiai RL, Acosta CJ, Danovaro-Holliday $M$, Baiqing D, Bhattacharya SK, Agtini MD, et al. A study of typhoid fever in five Asian countries: disease burden and implications for controls. Bull World Health Organ. 2008; 86: 260-268.

33. Crump JA, Luby SP, Mintz ED. The global burden of typhoid fever. Bull World Health Organ. 2004; 82: 346-353.

34. Abdoel TH, Pastoor R, Smits HL, Hatta M. Laboratory evaluation of a simple and rapid latex agglutination assay for the serodiagnosis of typhoid fever. Trans $R$ Soc Trop Med Hyg. 2007; 101(10): 1032-1038.

35. Krishnan $P$, Stalin $M$, Balasubramanian $S$. Changing trends in antimicrobial resistance of Salmonella enterica serovar typhi and Salmonella enterica serovar paratyphi A in Chennai. Indian J Pathol Microbiol. 2009; 52(4): 505.

36. Narayanappa D, Sripathi R, Jagdishkumar K, Rajani HS. Comparative study of dot enzyme immunoassay (Typhidot-M) and Widal test in the diagnosis of typhoid fever. Indian Pediatr. 2010; 47(4): 331-333.

37. Abucejo PE, Capeding MR, Lupisan SP, Arcay J, Sombrero LT, Ruutu P, Herva E. Blood culture confirmed typhoid fever in a provincial hospital in the Philippines. Southeast Asian J Trop Med Public Health. 2001; 32(3): 531-536.

38. Wain J, Hosoglu S. The laboratory diagnosis of enteric fever. J Infect Dev Ctries.2008; 2(06): 421-425.

39. Akoh JA. Relative sensitivity of blood and bone marrow cultures in typhoid fever. Trop Doct. 1991; 21(4): 174-176.

40. Mogasale V, Ramani E, Mogasale VV, Park J. What proportion of Salmonella Typhi cases are detected by blood culture? A systematic literature review. Ann Clin Microbiol Antimicrob. 2016; 15(1): 32. 
41. Gopalakrishnan V, Sekhar WY, Soo EH, Vinsent RA, Devi S. Typhoid fever in Kuala Lumpur and a comparative evaluation of two commercial diagnostic kits for the detection of antibodies to Salmonella Typhi. Singapore Med J. 2002; 43(7): 354-358.

42. Islam K, Sayeed MA, Hossen E, Khanam F, Charles RC, Andrews J, et al. Comparison of the performance of the TPTest, tubex, typhidot and Widal immunodiagnostic assays and blood cultures in detecting patients with typhoid fever in Bangladesh, including using a Bayesian latent class modelling approach. PLoS Negl Trop Dis. 2016; 10: e0004558.

43. Anusha R, Ganesh R, Lalitha J. Comparison of a rapid commercial test, Enterocheck WB ${ }^{\circledR}$, with automated blood culture for diagnosis of typhoid fever. Ann Trop Paediatr. 2011; 31(3): 231-234.

44. Sanjeev H, Nayak S, Asha PK, Rekha R, Karnaker $V$, Ganesh HR. A systematic evaluation of rapid dot-EIA, blood culture and Widal test in the diagnosis of typhoid fever. Nitte University $J$ Health Science. 2013; 3(1): 21.

45. Choudhury Z, Karim MR, Samad R, Islam S. Validity of immunochromatographic test for antibody in diagnosis of typhoid fever in children admitted in a tertiary care hospital. Chattagram Maa-O-Shishu Hosp Med Coll J. 2017; 16(2): 20-24.
46. Rahman $\mathrm{M}$, Siddique AK, Tam FCH, Sharmin S, Rashid $H$, lqbal $A$, et al. Rapid detection of early typhoid fever in endemic community children by the TUBEX ${ }^{\circledR}$ O9-antibody test. Diagn Microbiol Infect Dis. 2007; 58(3): 275281.

47. Naheed A, Ram PK, Brooks WA, Mintz ED, Hossain MA, Parsons MM, et al. Clinical value of Tubex ${ }^{\mathrm{TM}}$ and Typhidot ${ }^{\circledR}$ rapid diagnostic tests for typhoid fever in an urban community clinic in Bangladesh. Diagn Microbiol Infect Dis. 2008; 61(4): 381-386.

48. Prasad KJ, Oberoi JK, Goel N, Wattal C. Comparative evaluation of two rapid Salmonella-IgM tests and blood culture in the diagnosis of enteric fever. Indian J Med Microbiol. 2015; 33(2): 237.

49. Parveen S, Verma $P$, Dubey $K$, Verma MK. Comparative evaluation of ENTEROSCREENWBTM and Widal test in suspected cases of enteric fever. Int J Life Sci Scienti Res. 2015; 1(2): 84-88.

50. Ismail A. New advances in the diagnosis of typhoid and detection of typhoid carriers. Malays J Med Sci. 2000; 7(2): 3. 